\title{
Translation, cross-cultural adaptation, and measurement properties of the Nepali version of the central sensitization inventory (CSI)
}

\author{
Saurab Sharma ${ }^{1,2^{*}}$ D, Jyoti Jha ${ }^{1,3}$, Anupa Pathak ${ }^{2}$ and Randy Neblett ${ }^{4}$
}

\begin{abstract}
Background: Central sensitization is thought to be an important contributing factor in many chronic pain disorders. The Central Sensitization Inventory (CSI) is a patient-reported measure frequently used to assess symptoms related to central sensitization. The aims of the study were to translate and cross-culturally adapt the CSI into Nepali (CSI-NP) and assess its measurement properties.

Methods: The CSI was translated into Nepali using recommended guidelines. The CSI-NP was then administered on 100 Nepalese adults with sub-acute and chronic musculoskeletal pain with additional demographic and painrelated questions. The CSI-Nepali was administered again about 2 weeks later. Four measurement properties of the CSI-NP were evaluated: (1) internal consistency using Cronbach's alpha, (2) test-retest reliability using intraclass correlation coefficient $\left(\mathrm{ICC}_{2,1}\right)$, (3) measurement errors, and (4) construct validity testing five a priori hypotheses. Confirmation of construct validity was determined if a minimum of $75 \%$ of the hypotheses were met.

Results: The CSI was successfully translated into Nepali. Internal consistency and test-retest reliability were both excellent (Cronbach's alpha $=0.91$, and ICC $=0.98$ ). The standard error of measurement was 0.31 and the smallest detectable change was 0.86 . Four out of five (80\%) a priori hypotheses were met, confirming the construct validity: the CSI-NP correlated strongly with the Pain Catastrophizing Scale total scores $(r=0.50)$; moderately with the total number of pain descriptors ( $r=0.35)$; weakly with the Numerical Rating Scale $(r=0.25)$; and women had significantly higher CSI scores than men. However, the CSI scores did not correlate significantly with the total duration of pain, as hypothesized $(r=0.10)$.

Conclusions: The Nepali translation of the CSI demonstrated excellent reliability and construct validity in adults with musculoskeletal pain. It is now available to Nepali health care providers to help assess central sensitizationrelated signs and symptoms in individuals with musculoskeletal pain in research or clinical practice to advance the understanding of central sensitization in Nepalese samples.
\end{abstract}

Keywords: Pain, Musculoskeletal pain, Central nervous system sensitization, Cross-cultural comparison, Pain measurement, Nepal, Developing countries, Clinimetrics, Psychometric properties

\footnotetext{
* Correspondence: saurabsharma1@gmail.com

'Department of Physiotherapy, Kathmandu University of School of Medical Sciences, Dhulikhel, Nepal

${ }^{2}$ Centre for Musculoskeletal Outcomes Research, Otago Medical School, University of Otago, Dunedin, New Zealand

Full list of author information is available at the end of the article
}

(c) The Author(s). 2020 Open Access This article is licensed under a Creative Commons Attribution 4.0 International License, which permits use, sharing, adaptation, distribution and reproduction in any medium or format, as long as you give appropriate credit to the original author(s) and the source, provide a link to the Creative Commons licence, and indicate if changes were made. The images or other third party material in this article are included in the article's Creative Commons licence, unless indicated otherwise in a credit line to the material. If material is not included in the article's Creative Commons licence and your intended use is not permitted by statutory regulation or exceeds the permitted use, you will need to obtain permission directly from the copyright holder. To view a copy of this licence, visit http://creativecommons.org/licenses/by/4.0/ The Creative Commons Public Domain Dedication waiver (http://creativecommons.org/publicdomain/zero/1.0/) applies to the data made available in this article, unless otherwise stated in a credit line to the data. 


\section{Background}

Musculoskeletal pain is a highly prevalent condition. It is estimated that at least one in three persons experiences it [1]. It is one of the top reasons for years lived with disabilities in both developed and developing countries [2]. A musculoskeletal pain diagnosis increases the risk of mental health problems, other chronic illnesses, and all-cause mortality [3]. Being common in all age groups, including the working age groups, it possesses significant financial costs to both individuals and society [1]. Musculoskeletal pain is the leading cause of disability in Nepal [4] and is the number one reason for hospital admission [5]. Central sensitization (CS) is an important factor that is believed to contribute to many pain disorders, including musculoskeletal pain $[6,7]$.

CS involves the amplification of pain, and hypersensitivity to other environmental stimuli, within the central nervous system [8]. The Central Sensitization Inventory (CSI) is a relatively new patient-reported outcome measure used to assess somatic and emotional health-related symptoms that have been found to be associated with central sensitization [9]. A cut off score of 40 (out of a total possible score of 100) is often used to screen for the possible presence of central sensitization, so that diagnostic evaluation can be performed, and appropriate treatment can be initiated [10,11]. The original English version of the CSI, and translated versions in multiple other languages, have demonstrated good to excellent psychometric properties [9, 12-16]. The CSI total scores have been found to be associated with pain catastrophizing, pain intensity, pain interference, depression, anxiety, and quality of life [12, 17-19].

Pain is influenced by culture [20-22]. Therefore, translation, cross-cultural adaptation, and validation of the CSI in Nepali may benefit health care providers who provide assessment and treatment of Nepalese patient populations [23]. Furthermore, the availability of the CSI in Nepali would allow a new dimension of CS-related research in Nepal, which in turn, can contribute to understanding about CS from a population where CS has not been studied [24]. This could then be used in locally adapted pain education programs [25] using a previously proposed guide to improve patient outcomes in individuals with musculoskeletal pain [26].

Therefore, we aimed to translate and culturally adapt the CSI into Nepali (CSI-NP), using recommended guidelines [27], and to further evaluate its measurement properties, including internal consistency, test-retest reliability and construct validity (using hypothesis testing) with four CS-related clinical variables, including pain catastrophizing, pain intensity, duration of pain, and patient-reported pain descriptors. We hypothesized that the CSI-NP would demonstrate good to excellent internal consistency $[9,12,13,15,18]$, and excellent test- retest reliability $[9,12-15,18]$. We also hypothesized that CSI-NP would positively correlate with pain catastrophizing, pain intensity, and duration of pain $[12,17,18]$. In addition, because female gender has been found to be associated with CS-related disorders, we expected that women would have significantly higher CSI-NP scores compared to men [28-30]. Finally, we explored the association of CSI-NP scores with different types of pain descriptors (e.g., burning, aching, tingling), which, to our knowledge, has not been previously explored.

\section{Methods}

\section{Study design, and setting}

This research was conducted in two steps. In the first step, we performed a translation and cross-cultural adaptation of the CSI into Nepali. In the second step, we assessed the measurement properties of the Nepali version of the CSI. A longitudinal observational study design was used to evaluate the measurement properties of the CSI-NP. Data were collected from two sources: (1) the outpatient Physiotherapy Department of Dhulikhel Hospital, a tertiary care Hospital in Dhulikhel, Nepal, which is $30 \mathrm{~km}$ from Kathmandu, and (2) from the community of Bhaktapur, Kathmandu, Lalitpur, and Kavre districts from July to October in 2016.

A consecutive sampling method was used at the hospital, so that every patient presenting with musculoskeletal pain was invited to participate in the study, and those who met the inclusion criteria and consented to participate were included. A purposive sampling method was used to collect data from participants in the community. Participants were recruited door-to-door, and data were collected in their homes from the community sample. The protocol for this study was approved by the Institutional Review Committee of Kathmandu University School of Medical Sciences (ref number 76/16) before data collection began. Informed consent was obtained from every individual before administration of questionnaires. This study was a part of Bachelor of Physiotherapy degree thesis of the second author of this study. All data, including responses to CSI items, were collected in interview format to account for the high rate of illiteracy in Nepal [17].

\section{Participants}

A sample size of more than 50 participants is usually considered adequate for assessing measurement properties of a patient-reported outcome measure [31, 32]. Our goal was to recruit 100 participants for the assessment of the measurement properties in the study. The COSMIN (COnsensus-based Standards for the selection of health status Measurement INstruments) guidelines consider 100 participants "good" for the assessment of internal consistency, test-retest reliability, measurement errors, and construct validity [31]. 
For the evaluation of measurement properties portion of the study, a total of 115 individuals with self-reported and/or physician-diagnosed musculoskeletal pain were screened for eligibility. Eligible subjects were (1) able to understand and speak Nepali; (2) at least 18 years old; (3) having musculoskeletal pain for at least 1 month (sub-acute or chronic musculoskeletal pain); and (4) having a self-reported pain intensity of at least 3 out of 10 on a 11-point numeric pain rating scale for a minimum of 4 days in the past week [33]. Participants were excluded if they (1) had any recent history of trauma or fracture (within 6 weeks of data collection), (2) were diagnosed as having an acute illness (such as infection), malignancy, or diseases of the central nervous system, cardio-respiratory, gastrointestinal or urogenital system. Of the total sample of 115 subjects who were invited, five declined to participate and 10 were excluded because of the presence of acute pain associated with a neurological or cardiovascular disease, leaving 100 subjects for data analysis.

In addition, a sample of 20 individuals with sub-acute or chronic musculoskeletal pain was recruited from the outpatient Physiotherapy Department of Dhulikhel Hospital, Nepal for the pretesting and cognitive debriefing portion of this study. All study participants consented to participate. No participants were excluded based on low literacy skills.

\section{Step1: translation of the CSI into Nepali Initial translation process}

The original 25 items of the English version of the CSI Part A were translated into Nepali using recommended guidelines [27] using a similar approach to other Nepali translations of patient-reported outcome measures [34, 35]. First, two native Nepali translators (one with a medical background and one without), who were both fluent in English, translated the English version of the CSI into Nepali independently to produce two Nepali translations, T1 and T2. The two translations were synthesized into a single forward translation version (T3) which was facilitated by the first two authors. A written record of the synthesis, process with changes, and decisions was carefully documented. Some words, used to describe medical terms (example, pelvis area, jaw pain, sensitive towards bright light), were either unavailable in Nepali as single words or phrases, or would not be understood by most Nepalese, so a number of experts were consulted, including dentists, gynaecologists, and opthalmologists. Two of the original developers of the CSI were also contacted to clarify the meaning of some of these words in the original English version of the CSI. Finally, the research team pooled all of this information into a common single synthesized version of the CSI, which was used for the back translation into English.
The translation of the Part B of the CSI was deemed "untranslatable" during the preliminary "translatability assessment" of the scale. Translatability assessment is an important pre-requisite of translation and cross-cultural adaptation of outcome measures [36, 37]. This is because Part B of the CSI has names of medical diagnoses that have no comparable Nepali words or translations, are less commonly studied [24] or used in clinical practice in Nepal, and are likely not recognizable by most Nepali-speaking patients. Therefore, a single forward translation of the Part B was performed and it was not subjected to later phases of translations including backtranslation or expert committee meeting discussions. All English names of the medical diagnoses were re-written in Devanagari script (i.e. Nepali script).

\section{Back translation into English}

A translator without a medical background, who was blind to the original English version of the CSI, translated the synthesized Nepali translation (T4) back into English.

\section{Expert committee meeting and review}

An expert committee was comprised of the researchers and the translators involved in the translation processes. All translation versions were discussed in this meeting, and after the consensus from the committee members, a pre-final Nepali version of the CSI (T5) was created with minimal modification on the choices of words and sentence structure on the T3 version. The English backtranslation of this version was then sent to two of the original CSI developers (author RN and Prof Robert Gatchel) for review of the items. Minor changes were made in some items after suggestions from the developers, resulting in a T6 version, which was used for pretesting.

\section{Pretesting and cognitive debriefing}

The Nepali version of the CSI was tested on 20 individuals with sub-acute or chronic musculoskeletal pain, representing different age categories, both sexes, and a variety of education levels. Guidelines recommend from five to 30 participants adequate for pre-testing and cognitive debriefing $[27,38]$. We retrieved no new information after testing 20 participants during the in-depth cognitive interviews. Participants were requested to complete the questionnaire by themselves if they could read and write. The second author (JJ) administered the questionnaire in an interview format for participants who had difficulty reading and writing. All participants were asked to clarify the meaning of the instructions, items, and response options, to assure comprehensibility of the questionnaire for content validity of the CSI [39, 40]. Semantic equivalence was assured in this final version. Minor changes in sentence structure and grammar were made after pretesting and cognitive debriefing, 
resulting in a final version of the CSI-Nepali (CSI-NP), which can be found in the online only Supplement 1 . The original English version of the CSI is available as online Supplement 2.

\section{Step 2: assessment of measurement properties}

After the translation and pre-testing processes were completed, data were collected for assessment of the measurement properties of the CSI-NP. We followed the methodological quality, proposed by the COSMIN recommendations [41]. To account for the low literacy rates in Nepal, all demographic and clinical data were collected by physiotherapists or physiotherapy students in an interview format [42]. The CSI items were also read aloud to each participant, and their answers were recorded by the interviewer. The CSI-NP was readministered at an interval of approximately 2 weeks for the assessment of test-retest reliability. All 100 participants completed the retest measurement. During the follow-up assessment, participants from the community were interviewed face-to-face, and those recruited from the hospital were interviewed via phone calls.

\section{Measures used \\ Demographic characteristics}

Information regarding participants' age, gender, marital status, religion, ethnicity, education and occupation were collected to describe the participant characteristics.

\section{Assessment of pain}

Duration of pain was recorded as the number of weeks since the onset of current pain. Pain intensity was assessed using the Nepali version of the 11-point $\mathrm{Nu}$ merical Rating Scale (NRS), which has been shown to be valid and reliable, with excellent test-retest reliability (Intraclass correlation coefficient, ICC $=0.81$ ) [43]. Three measures of pain intensity were assessed, including current pain, best pain, and worst pain in the last 24 $h$ [43]. We averaged the three scores into one pain intensity variable, as was done previously in the validation paper of the Nepali version of the NRS [43]. The NRS scores range from "0" = "No pain" to "10" = "Extreme pain", where a greater score indicates more intense pain.

Quality of pain was assessed with a list of descriptors, which we previously identified as the most common pain quality descriptors in Nepal [44]. These descriptors are similar to the revised short form of the McGill Pain Questionnaire [45]. Specific descriptors included "burning, tingling, piercing, heavy, numb, cramping, stretching, aching, and infection-like." Participants reported "present" or "absent" on each descriptor. Frequency of their occurrence was also reported. In addition, participants were given the opportunity to provide additional words to describe their pain.
Sites of pain were assessed using a pain diagram. Participants were asked to mark their painful sites on a body chart. The sites of pain were categorized as pain in the neck, shoulder and arm, elbow and forearm, wrist and hand, upper back, lower back, hip and thighs, knee and leg, and ankle and foot.

\section{Pain Catastrophizing scale}

Pain catastrophizing was assessed using the Nepali version of 13-item Pain Catastrophizing Scale (PCS) [33]. Each item is scored on a Likert scale with "0" = "Never" and "4" = "Always." The total score ranges from 0 to 52 , with higher scores indicating more pain catastrophizing. The Nepali version of the PCS is a valid and reliable measure with good to excellent internal consistency (Cronbach's alpha $=0.83$ 0.93 ) and excellent test-retest reliability (ICC $=0.88-0.90$ ) [33].

\section{Central sensitization inventory}

Part A of the CSI [9] includes 25-items on a Likert scale, scored from "0" = "Never" to "4" = "Always," with a total score range of 0 to 100 . Higher scores indicate higher central sensitization related symptoms. Five severity level score ranges are available to aid with clinical interpretation (subclinical $=0$ to 29 ; mild $=30$ to 39 ; moderate $=40$ to 49 ; severe $=50$ to 59 ; and extreme $=60$ to 100) [46]. Part B, which is not scored, asks if one has been previously diagnosed with a list of common central sensitizationrelated diagnoses. Part B was not used in the phase 2 of this study.

\section{Statistical analyses}

The raw data were entered into SPSS 24.0 for analyses, and descriptive statistics were computed for demographic and pain variables.

\section{Reliability}

Internal consistency The internal consistency of the CSI-NP was computed using Cronbach's alpha. We considered Cronbach's alpha between $0.80-0.89$ to be good, and values equal to 0.90 or more as excellent internal consistency [47].

Test-retest reliability The Intraclass Correlation Coefficient $\left(\mathrm{ICC}_{2,1}\right)$ was used to compute the two-week testretest reliability using two way mixed model and absolute agreement. The ICC value of more than 0.75 was considered to be excellent test-retest reliability [47].

\section{Measurement error}

Standard error of measurement (SEM) The SEM was calculated initially by using the formula, $\mathrm{SEM}=\mathrm{SD}$ * 
$\sqrt{ }(1-\mathrm{ICC})$, where $\mathrm{SD}=$ standard deviation of change of CSI-NP from baseline to follow-up.

Smallest detectable change (SDC) The SDC for 95\% confidence interval was computed using the formula $\mathrm{SEM} \times \mathrm{Z} \times \sqrt{2}$; where $\mathrm{Z}$ is the $\mathrm{Z}$ value for the $95 \% \mathrm{CI}$ and $\sqrt{ } 2$ is used to account for the variance of two measurements [48].

Bland-Altman plot The Bland-Altman Plot was created to visualize systematic errors in the baseline and final time-point scores of the CSI [49].

\section{Construct validity}

Hypothesis testing As indicated earlier, a total of five hypotheses were tested to evaluate the construct validity of the CSI-NP, using a standard hypothesis testing methodology $[31,50]$. Spearman correlation coefficient was used to assess the association of the CSI-NP with the PCS, the NRS, duration of pain, and the total number of pain descriptors. We considered $r$ values of less than 0.30 as a weak correlation, $0.30-0.49$ as a moderate correlation and values of 0.50 or higher as a strong correlation [51]. An unpaired $t$-test was used to assess if women had significantly higher CSI score than men. The results are reported as "hypothesis confirmed" or "hypothesis not confirmed". The total number of met hypotheses were added and reported as percentages. If the total percentage was more than $75 \%$, we confirmed the construct validity of the CSI-NP as per the COSMIN recommendations [32].

\section{Results}

\section{Translation and cross-cultural adaptation}

Three items required cross-cultural adaptation during the translation phase, with input from two of the developers of the CSI. Item 19, "I have pain in my jaw," was difficult to translate because a single Nepali word for "jaw" does not exist. After discussion within the translation team and a dentist, the item was translated as "I have pain in the muscles and joints around my molar teeth." Item 25, "I have pain in my pelvic area," was translated as "I have pain between my hips (near my private body parts)" because there was no agreement on a Nepali word for pelvic area that is easily understandable. Finally, the Nepali translation of item 24, "I suffered trauma as a child," only reflected physical trauma in the initial Nepali translation. Therefore, to assure the conceptual equivalence we explicitly added "physical or psychological" before "trauma" to clarify that trauma could be either physical or psychological. Translation of the part B of the CSI was difficult because Nepali words for most of the medical conditions were not available in
Nepali, therefore the vigorous forward and backward translation of part B of the CSI was not performed.

\section{Demographic and pain characteristics}

The mean age of the study participants was 42.01 (14.61 $\mathrm{SD})$ years. The majority of participants were female (67\%), married (76\%), and Hindu by religion (79\%). Almost half of the participants were unemployed (47\%). The mean duration of pain was 111 weeks, the median was 24 weeks, and the range was 4 to 1280 weeks. The most common sites of pain were the knee (32\%) followed by the low back (24\%). Additional socio-demographic details are presented in Table 1.

Total mean score for the CSI-NP was 22.85 (SD 12.35) with scores ranging from 0 to 59. A majority of the sample $(75 \%)$ scored in the subclinical severity range (scores $<29$ ). Only $14 \%$ were categorized as mild, $8 \%$ as moderate, and $3 \%$ as severe central sensitization related symptoms. No subjects scored in the extreme category. The total mean score for the PCS was 18.51 (SD 11.84; range $0-52$ ) and the NRS was 5.17 (SD 1.85, range 2-10).

\section{Reliability}

Internal consistency of the CSI-NP was good (Cronbach's alpha $=0.87$ ). Test-retest reliability of the CSI-NP was excellent with ICC $=0.98$ (95\% CI: 0.97, 0.99).

\section{Measurement error}

Standard error of measurement was 0.31. Smallest detectable change (SDC) was 0.86. The Bland-Altman Plot, shown in Fig. 1, presents the distribution and variability in the CSI scores in the baseline and final measurements for each study participant.

\section{Construct validity}

As shown in Table 2, a total of 4 of 5 a priori hypotheses (80\%) were met, confirming the construct validity of the CSI-NP. The CSI-NP correlated strongly with PCS with $(r=0.50, P<0.001)$, moderately with the total number of pain descriptors $(r=0.35, P<0.001)$, and weakly with NRS pain intensity $(r=0.25, P=0.013)$. Mean CSI-NP scores were significantly higher in women (25.21; SD 12.23) compared to the men (18.06; SD 10.87). The correlation of the CSI-NP with pain duration $(r=0.10 ; P=$ $0.315)$ was weak and non-significant.

Exploratory analysis revealed that CSI-NP scores were frequently and significantly associated with three pain descriptors, including heavy, tingling, and infection-like pain (throbbing pain), but not with other types of pain, including achy, piercing, stretching, numb, cramping, and burning (see Table 3). 
Table 1 Socio-demographic characteristics with distribution of site of pain

\begin{tabular}{|c|c|}
\hline Variables & Frequency N (\%) \\
\hline \multicolumn{2}{|l|}{ Sex } \\
\hline Men & $33(33 \%)$ \\
\hline Women & $67(67 \%)$ \\
\hline \multicolumn{2}{|l|}{ Marital status } \\
\hline Married & $76(76 \%)$ \\
\hline Single & $16(16 \%)$ \\
\hline Separated & $1(1 \%)$ \\
\hline Missing & $7(7 \%)$ \\
\hline \multicolumn{2}{|l|}{ Religion } \\
\hline Hindu & 79 (79\%) \\
\hline Buddhist & $11(11 \%)$ \\
\hline Others & $3(3 \%)$ \\
\hline Missing & $7(7 \%)$ \\
\hline \multicolumn{2}{|l|}{ Ethnicity } \\
\hline Brahmin & $31(31 \%)$ \\
\hline Newar & $28(28 \%)$ \\
\hline Chettri & $18(18 \%)$ \\
\hline Tamang & $9(9 \%)$ \\
\hline Others & $7(7 \%)$ \\
\hline Missing & $7(7 \%)$ \\
\hline \multicolumn{2}{|l|}{ Occupation } \\
\hline Unemployed & 47 (47\%) \\
\hline Office & $20(20 \%)$ \\
\hline Agriculture & $11(11 \%)$ \\
\hline Student & $8(8 \%)$ \\
\hline Others & $7(7 \%)$ \\
\hline Missing data & $7(7 \%)$ \\
\hline \multicolumn{2}{|l|}{ Education } \\
\hline Never went to school & $30(30 \%)$ \\
\hline Less than 5 years & $15(15 \%)$ \\
\hline $6-12$ years & $20(20 \%)$ \\
\hline Bachelor and above & $28(28 \%)$ \\
\hline Missing data & $7(7 \%)$ \\
\hline \multicolumn{2}{|l|}{ Site of pain } \\
\hline Knee and leg & $32(32 \%)$ \\
\hline Low back & $24(24 \%)$ \\
\hline Two or more pain sites & $16(16 \%)$ \\
\hline Upper back & $7(7 \%)$ \\
\hline Ankle and Foot & $6(6 \%)$ \\
\hline Shoulder & $5(5 \%)$ \\
\hline Neck & $4(4 \%)$ \\
\hline Wrist & $4(4 \%)$ \\
\hline Hip and thigh & $2(2 \%)$ \\
\hline
\end{tabular}

\section{Discussion}

The study aimed to translate and cross-culturally adapt the CSI into Nepali and evaluate its measurement properties. The Nepali translation of the CSI was shown to be a reliable and a valid measure with small measurement errors. In general, the CSI scores for the Nepalese sample were lower compared to samples collected from other geographic regions and other pain populations $[9,13]$. In fact, the mean CSI-NP score in our clinical sample (22.85) was smaller than healthy control samples from the USA (28.90) [9] and Brazil (37.14) [12]. Mean CSI scores have varied in other clinical samples (with individuals with fibromyalgia generally scoring the highest and those with localized pain scoring lower) and with different cultural regions (with generally higher scores in Western countries and generally lower scores in Eastern countries) [19]. For instance, a Japanese chronic pain sample scored in a similar range as our clinical sample (21.91) [18]. Perhaps there are cultural differences in the perception and reporting of central sensitization symptoms. Future studies may want to concurrently collect data on CSI between two or more countries or cultures using the identical study designs in order to determine the true differences in the CSI scores across countries or cultures as we previously recommended [52].

\section{Reliability}

We found excellent reliability (internal consistency and test-retest reliability of the CSI-NP consistent with multiple language versions of the CSI $[9,12-15,18,53]$. The internal consistency of the CSI-NP was comparable to other language versions, whereas, the test-retest reliability was larger than for other language versions including the Brazilian Portuguese, Dutch, English, French, Serbian, and Spanish (ICC $=0.82$ to 0.95$)[9,12-15,53]$ but comparable to Greek and Gujrati versions [54, 55]. The primary reason for a higher test-retest reliability may be that the researcher who administered the follow-up CSI measure provided the values of the baseline measurement to each participant. This could have confounded the follow-up scores, yielding high levels reliability, as reflected in the Bland-Altman Plot in Fig. 1. Also, unlike other CSI studies, the CSI in the present study was administered in an interview format. Future studies could explore if there are differences in test-retest reliability of the CSI-NP in a self-reported format. We were unable to analyze reliability separately for face-to-face and telephone interviews. In a previous and similar validation study, we found no differences in test-retest reliability of the Nepali Disability of Arm, Shoulder, and Hand Questionnaire [56]. Further studies may assess the reliability separately for face-to-face administration of CSI-NP.

\section{Measurement error}

Measurement errors, although important measurement property of a patient-reported outcome measure, is rarely 


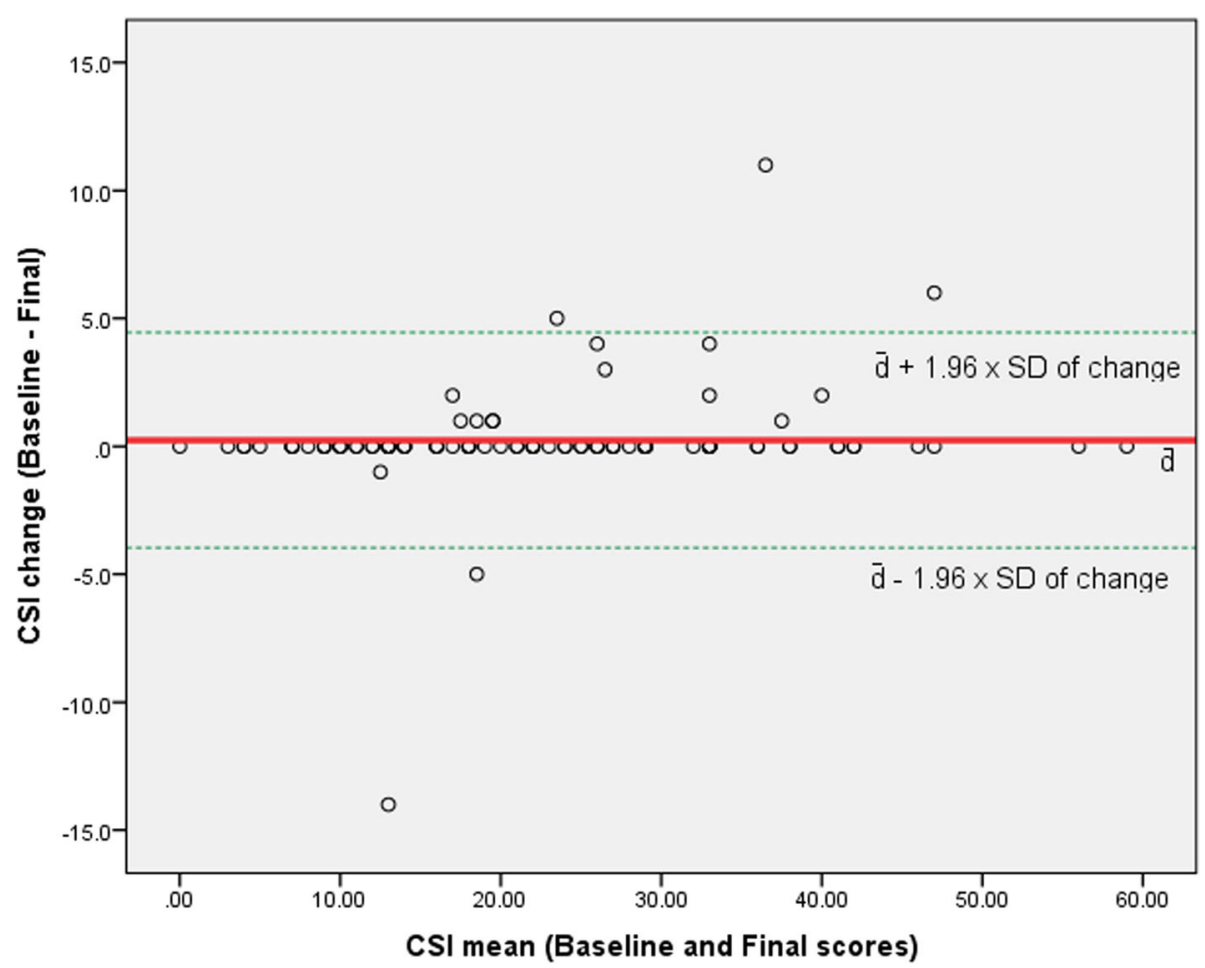

Fig. 1 Bland-Altman Plot for the Nepali Central Sensitization Inventory. Note: Y-axis is the change of the CSI scores and X-axis is the mean of the CSI scores at the baseline and final measurements. Solid red line is the mean change of score ( $\bar{d})$; and dotted green lines are $\bar{d} \pm Z \times S_{\text {change }}$ (where $\mathrm{Z}=1.96$ for $95 \%$ confidence interval)

measured in the CSI studies. The CSI-NP showed standard error of measurement $(\mathrm{SEM}=0.86)$ smaller than other language versions $(\mathrm{SEM}=1.84$ to 3.16$)[13,14,54,55]$. The SDC $(=8.86)$ in the current study was also smaller than two previous studies (SDC $=5.90$ and 7.83$)[13,55]$. It could be because of the same reasons that accounted for high test-retest reliability as described above. Based on the current findings, any change score more than 1 unit (out of 100) may be viewed as true change for the Nepali version of the CSI. Future studies should explore measurement errors for other language versions of the CSI and compare against minimum important change scores, which should ideally exceed the SDC values $[31,57]$. The assessment of measurement error should be repeated in other Nepalese samples without disclosing the baseline scores to the patients during the retest assessment, which will potentially provide more accurate estimate of measurement errors.

Table 2 Results of hypotheses testing for construct validity of the CSI-NP $(N=100)$

\begin{tabular}{|c|c|c|c|}
\hline \multirow[t]{2}{*}{ Scale } & \multicolumn{3}{|l|}{ Construct validity (correlation with baseline scores) } \\
\hline & Hypothesis & Results & $\begin{array}{l}\text { Hypothesis } \\
\text { confirmed? }\end{array}$ \\
\hline \multirow[t]{5}{*}{$\begin{array}{l}\text { CSI- } \\
\text { NP }\end{array}$} & $\begin{array}{l}\text { Positive weak to strong associations with pain } \\
\text { intensity }\end{array}$ & $r=0.25(P=0.013)$ & Yes \\
\hline & $\begin{array}{l}\text { Positive weak to strong associations with pain } \\
\text { catastrophizing }\end{array}$ & $r=0.50(P<0.001)$ & Yes \\
\hline & $\begin{array}{l}\text { Positive weak to moderate associations with } \\
\text { duration of pain }\end{array}$ & $r=0.10(P=0.315)$ & No \\
\hline & $\begin{array}{l}\text { Positive weak to moderate associations with total } \\
\text { number of types of pain }\end{array}$ & $r=0.35(P<0.001)$ & Yes \\
\hline & $\begin{array}{l}\text { Women would have significantly higher CSI scores } \\
\text { than men }\end{array}$ & $\begin{array}{l}\text { Women had significantly higher CSI scores than men [Mean difference }= \\
7.15(95 \% \mathrm{Cl}: 2.11,12.19) ; P=0.005]\end{array}$ & Yes \\
\hline
\end{tabular}

Total hypothesis met

4 of $5(80 \%)$ 
Table 3 Pain quality and their correlation with the CSI-NP ( $N=$ 100)

\begin{tabular}{llll}
\hline Pain quality & $\mathrm{N}$ & $\boldsymbol{r}$ with CSI-NP & $\boldsymbol{P}$ \\
\hline Kat-kat (Achy) & 64 & 0.20 & 0.052 \\
Piercing pain & 57 & 0.05 & 0.587 \\
Heavy pain & 52 & $\mathbf{0 . 2 2}^{\mathbf{a}}$ & $\mathbf{0 . 0 3 2}$ \\
Stretching pain & 40 & 0.11 & 0.300 \\
Tingling & 39 & $\mathbf{0 . 2 2 ^ { \mathbf { a } }}$ & $\mathbf{0 . 0 2 7}$ \\
Numb & 37 & 0.19 & 0.065 \\
Cramping pain & 30 & 0.19 & 0.063 \\
Infection-like pain (throbbing) & 28 & $\mathbf{0 . 2 2 ^ { \mathbf { a } }}$ & $\mathbf{0 . 0 2 6}$ \\
Burning & 26 & 0.16 & 0.103 \\
\hline
\end{tabular}

Abbreviations. N, total number of participants reporting "Yes" on the specific quality of pain; $r$, correlation coefficient

andicates significance level at $<0.05$. Significant associations with the CSI-NP are highlighted in bold text

\section{Construct validity}

Eighty percentage (four of five) of a priori hypotheses were met, which support the construct validity of the CSI-NP based on criteria proposed by Terwee and colleagues [32]. Construct validity of the CSI has primarily been assessed using factor analyses in previous studies. The form of hypothesis testing used in the present study is another recommended approach $[31,50]$ for assessing the construct validity of a patient-reported outcome measures.

The hypotheses were made based on the direction and magnitude of associations. Due to lesser number of similar associations being tested in previous research, the magnitude of association hypothesized were open wider. Although the hypotheses on the association of the CSI-NP with pain intensity, pain catastrophizing were met as hypothesized, the strength of correlations we found are varied. For example, the correlation of the CSI-NP with pain intensity $(\mathrm{r}=0.25)$ was lower than the Dutch and Japanese versions ( $r=0.51$ and 0.42 respectively) $[15,18]$. Similarly, correlation of the CSI-NP with pain catastrophizing $(r=$ $0.50)$ was similar to the Dutch version $(r=0.52)$ but lesser than Brazilian Portuguese version $(r=0.68)$. Our hypothesis that women experience more CS related symptoms is consistent with previous literature [28-30]. The a priori hypothesis that the CSI scores would positively be associated with total number of types of pain was met, which provides an indication that individuals with higher CSI scores are more likely to report more number of types of pain (e.g., achy, heavy, tingling, throbbing). If this finding is consistently replicated, it could stand as an important feature of CS that can easily be identified during communication with patients. Although CS is expected to be associated to chronicity, we did not find a significant association between the CSI-NP and total duration of pain. The association of the CSI with the duration of pain and total number of types of pain need further exploration.
Exploratory analyses for the association of CSI scores with heavy pain, tingling pain, and throbbing pain is interesting, and should be confirmed in further studies to assess if particular types of pain are associated more strongly with central sensitization signs and symptoms than the other types of pain. If this is the case, type of pain then could help flag central sensitization related signs and symptoms.

\section{Strengths and limitations}

Despite the methodological strengths, i.e. adoption of standard recommended guidelines to translate and crossculturally adapt CSI into Nepali [27], and compliance with COSMIN recommendations $[31,41]$ to perform analyses for measurement properties, the limitations of the study should be carefully considered. First, we did not perform a factor analysis of the CSI-NP in the current study because 150 additional participants would have been required based on recommended guidelines (with 10 participants per item [31]). Because of the feasibility reasons, it was not practical to collect an additional 150 subjects simply for the purpose of a factor analysis. Second, we did not perform a responsiveness analysis and the minimum important change scores for the CSI-NP. Future studies might consider the assessment of responsiveness to change and minimum important change scores to be able to better interpret the change of the CSI-NP scores after interventions to target central sensitization in the individuals with musculoskeletal pain. Third, we did not assure that the patients were unchanged between the test and re-test interval for the assessment of test-retest reliability and measurement errors, as recommended by the COSMIN guidelines [31, 41]. It was our assumption that a duration of 2 weeks was not enough time for any significant change to occur in the construct of central sensitization in all or most of the study participants. Fourth, the CSI-NP was tested only on adults with musculoskeletal pain, therefore, the questionnaire should not be assumed valid for other populations (e.g., pediatric patients and patients with cancer-related pain). Fifth, we did not administer the CSI on normal healthy individuals to explore if the CSI scores discriminated individuals with and without pain and if the scores would differ from individuals with chronic pain syndromes. Future studies should consider the assessment of the CSI scores in normal healthy individuals to see how the CSI scores differ between samples with or without pain. Finally, because of non-random sampling methods used to recruit participants from the community, two thirds of the study participants recruited in the current sample were women. The readers should be wary of this when considering the generalizability of the study findings. The future studies may consider random sampling approaches to recruit study participants from community settings. 


\section{Conclusions}

The CSI was successfully translated into Nepali for the first time. The CSI scores were shown to be reliable and valid and that it can be used to assess signs and symptoms related to central sensitization in adults with musculoskeletal pain in Nepal in clinical practice or research. Future studies on the CSI-NP should assess treatment responsiveness, compute a minimum important change score, compare if that score is larger than the values of measurement errors, and validate the CSI in other pain populations to extend its clinical and research use.

\section{Supplementary information}

Supplementary information accompanies this paper at https://doi.org/10. 1186/s12883-020-01867-1.

Additional file 1. Nepali-CSI final version.

Additional file 2. CSI English version.

\section{Abbreviations}

Cl: Confidence Interval; COSMIN: COnsensus-based Standards for the selection of health status Measurement INstruments; CS: Central Sensitization; CSI: Central Sensitization Inventory; CSI-NP: The Nepali version of the Central Sensitization Inventory; ICC: Intraclass Correlation Coefficient; NRS: Numerical Rating Scale; PCS: Pain Catastrophizing Scale; SD: Standard Deviation; SDC: Smallest Detectable Change; SEM: Standard Error of Measurement; SPSS: Statistical Package for Social Sciences

\section{Acknowledgements}

The authors would like to thank everyone involved in the translation of the CSI and all study participants who volunteered to participate in this study. The authors would also like to thank Professor Robert J. Gatchel for his contribution to the translation process of the CSI.

\section{Authors' contributions}

SS conceived the study. JJ collected data. AP contributed in the recruitment of participants. SS, JJ, AP, RN contributed in the translation process of the CSI and input on the study protocol. SS performed all analyses with assistance from AP. SS drafted the first manuscript. RN, AP, JJ provided input in the interpretation of the results, read, revised the manuscript. All authors read and approved the final version of the paper.

\section{Funding}

None to declare.

\section{Availability of data and materials}

The dataset used and/or analysed during the current study is available from the corresponding author on reasonable request.

\section{Ethics approval and consent to participate}

The study was approved by the Institutional Review Committee of Kathmandu University School of Medical Sciences, Dhulikhel Nepal. All study participants provided informed consent before data collection. Participants who can read and sign the consent form provided a written consent. The participants who could not sign provided verbal consent and a witness signed on their behalf.

\section{Consent for publication}

Not required.

\section{Competing interests}

None.

\section{Author details}

'Department of Physiotherapy, Kathmandu University of School of Medical Sciences, Dhulikhel, Nepal. ${ }^{2}$ Centre for Musculoskeletal Outcomes Research,
Otago Medical School, University of Otago, Dunedin, New Zealand. ${ }^{3}$ Department of Physiotherapy, Grande International Hospital, Kathmandu, Nepal. ${ }^{4}$ PRIDE Research Foundation, Dallas, TX, USA.

Received: 23 January 2020 Accepted: 21 July 2020

Published online: 27 July 2020

\section{References}

1. Briggs AM, Woolf AD, Dreinhöfer K, Homb N, Hoy DG, Kopansky-Giles D, Åkessong K, March L. Reducing the global burden of musculoskeletal conditions. Bull World Health Organ. 2018;96(5):366-8.

2. GBD 2016 Disease and Injury Incidence and Prevalence Collaborators. Global, regional, and national incidence, prevalence, and years lived with disability for 328 diseases and injuries for 195 countries, 1990-2016: a systematic analysis for the global burden of disease study 2016. Lancet. 2017;390(10100):1211-59.

3. Briggs AM, Cross MJ, Hoy DG, Sànchez-Riera L, Blyth FM, Woolf AD, March L. Musculoskeletal health conditions represent a global threat to healthy aging: a report for the 2015 World Health Organization world report on ageing and health. Gerontologist. 2016;56(Suppl_2):S243-55.

4. James SL, Abate D, Abate KH, Abay SM, Abbafati C, Abbasi N, Abbastabar H, AbdAllah F, Abdela J, Abdelalim A, et al. Global, regional, and national incidence, prevalence, and years lived with disability for 354 diseases and injuries for 195 countries and territories, 1990\&\#x2013;2017: a systematic analysis for the Global Burden of Disease Study 2017. Lancet. 2018:392(10159):1789-858.

5. Sürmeli A, Tolunay T, Yasin Y, Kusoglu H, Arcan A, Jaiswal A, Gökmen B, Acharya B, Arslan B, Baka BE et al: Child health, parasites and lower socioeconomic status: outcomes of a long-term screening, intervention and training study by health volunteers in rural Nepal. Acta Trop. 2019;202: 105263.

6. Harte SE, Harris RE, Clauw DJ. The neurobiology of central sensitization. J Appl Behav Res. 2018;23(2):e12137.

7. Pelletier R, Higgins J, Bourbonnais D. Is neuroplasticity in the central nervous system the missing link to our understanding of chronic musculoskeletal disorders? BMC Musculoskelet Disord. 2015;16(1):25.

8. Woolf CJ. Central sensitization: implications for the diagnosis and treatment of pain. Pain. 2011:152(3 Suppl):S2-15.

9. Mayer TG, Neblett R, Cohen H, Howard KJ, Choi YH, Williams MJ, Perez Y, Gatchel RJ. The development and psychometric validation of the central sensitization inventory. Pain Pract. 2012;12(4):276-85.

10. Nijs J, Apeldoorn A, Hallegraeff H, Clark J, Smeets R, Malfliet A, Girbes EL, De Kooning M, Ickmans K. Low back pain: guidelines for the clinical classification of predominant neuropathic, nociceptive, or central sensitization pain. Pain Physician. 2015;18(3):E333-46.

11. Neblett R, Cohen H, Choi Y, Hartzell MM, Williams M, Mayer TG, Gatchel RJ. The central sensitization inventory (CSI): establishing clinically significant values for identifying central sensitivity syndromes in an outpatient chronic pain sample. J Pain. 2013:14(5):438-45.

12. Caumo W, Antunes LC, Elkfury JL, Herbstrith EG, Sipmann RB, Souza A, Torres IL, dos Santos VS, Neblett R. The central sensitization inventory validated and adapted for a Brazilian population: psychometric properties and its relationship with brain-derived neurotrophic factor. J Pain Res. 2017;10:2109-22.

13. Cuesta-Vargas Al, Roldan-Jimenez C, Neblett R, Gatchel RJ. Cross-cultural adaptation and validity of the Spanish central sensitization inventory. Springerplus. 2016;5(1):1837.

14. Knezevic A, Neblett R, Jeremic-Knezevic M, Tomasevic-Todorovic S, Boskovic K, Colovic P, Cuesta-Vargas A. Cross-cultural adaptation and psychometric validation of the Serbian version of the central sensitization inventory. Pain Pract. 2018:18(4):463-72.

15. Kregel J, Vuijk PJ, Descheemaeker F, Keizer D, van der Noord R, Nijs J, Cagnie $B$, Meeus $M$, van Wilgen $P$. The Dutch central sensitization inventory (CSI): factor analysis, discriminative power, and test-retest reliability. Clin J Pain. 2016;32(7):624-30.

16. Scerbo T, Colasurdo J, Dunn S, Unger J, Nijs J, Cook C. Measurement properties of the central sensitization inventory: a systematic review. Pain Pract. 2018:18(4):544-54

17. Huysmans E, Ickmans K, Van Dyck D, Nijs J, Gidron Y, Roussel N, Polli A, Moens M, Goudman L, De Kooning M. Association between symptoms of central sensitization and cognitive behavioral factors in people with chronic nonspecific low Back pain: a Cross-sectional study. J Manip Physiol Ther. 2018;41(2):92-101. 
18. Tanaka K, Nishigami T, Mibu A, Manfuku M, Yono S, Shinohara Y, Tanabe A, Ono R. Validation of the Japanese version of the central sensitization inventory in patients with musculoskeletal disorders. PLoS One. 2017;12(12):e0188719.

19. Neblett R. The central sensitization inventory: a user's manual. J Appl Biobehav Res. 2018;23(2):e12123.

20. Ferreira-Valente A, Sharma S, Torres S, Smothers Z, Pais-Ribeiro J, Abbott JH, Jensen MP. Does religiosity/spirituality play a role in function, pain-related beliefs, and coping in patients with chronic pain? A Systematic Review. J Relig Health. 2019.

21. Sharma S, Abbott JH, Jensen MP. Why clinicians should consider the role of culture in chronic pain. Braz J Phys Ther. 2018;22(5):345-6.

22. Sharma S, Ferreira-Valente A, de CWAC AJH, Pais-Ribeiro J, Jensen MP. Group Differences Between Countries and Between Languages in PainRelated Beliefs, Coping, and Catastrophizing in Chronic Pain: A Systematic Review. Pain Med. 2020:pnz373.

23. Sharma S, Jensen MP. Cross-cultural adaptations of patient-reported outcome measures can be very useful. Ann Phys Rehabil Med. 2019

24. Sharma S, Jensen MP, Pathak A, Sharma S, Pokharel M, Abbott JH. State of clinical pain research in Nepal: a systematic scoping review. PAIN Reports. 2019;4(6):e788

25. Sharma S, Jensen MP, Moseley GL, Abbott JH. Results of a feasibility randomised clinical trial on pain education for low back pain in Nepal: the pain education in Nepal-low Back pain (PEN-LBP) feasibility trial. BMJ Open. 2019;9(3):e026874

26. Nijs J, Paul van Wilgen C, van Oosterwijck J, van Ittersum M, Meeus M. How to explain central sensitization to patients with 'unexplained' chronic musculoskeletal pain: practice guidelines. Man Ther. 2011;16(5):413-8.

27. Beaton DE, Bombardier C, Guillemin F, Ferraz MB. Guidelines for the process of cross-cultural adaptation of self-report measures. Spine (Phila Pa 1976). 2000;25(24):3186-91.

28. Voss U, Lewerenz A, Nieber K. Treatment of irritable bowel syndrome: sex and gender specific aspects. Handb Exp Pharmacol. 2012;214:473-97.

29. Howard KJ, Mayer TG, Neblett R, Perez Y, Cohen H, Gatchel RJ. Fibromyalgia syndrome in chronic disabling occupational musculoskeletal disorders: prevalence, risk factors, and posttreatment outcomes. J Occup Environ Med. 2010;52(12):1186-91.

30. Shyti $R$, de Vries B, van den Maagdenberg A. Migraine genes and the relation to gender. Headache. 2011;51(6):880-90.

31. Mokkink LB, Terwee CB, Patrick DL, Alonso J, Stratford PW, Knol DL, Bouter LM, CW de Vet HCW: COSMIN Checklist Manual. 2012. http://fac.ksu.edu.sa/ sites/default/files/cosmin_checklist_manual_v9.pdf.

32. Terwee $\mathrm{CB}$, Bot $\mathrm{SD}$, de Boer MR, van der Windt DA, Knol DL, Dekker J, Bouter LM, de Vet HC. Quality criteria were proposed for measurement properties of health status questionnaires. J Clin Epidemiol. 2007;60(1):34-42.

33. Sharma S, Thibault P, Abbott JH, Jensen MP. Clinimetric properties of the Nepali version of the pain Catastrophizing scale in individuals with chronic pain. J Pain Res. 2018;11:265-76.

34. Sharma S, Pathak A, Abbott JH, Jensen MP. Measurement properties of the Nepali version of the Connor Davidson resilience scales in individuals with chronic pain. Health Qual Life Outcomes. 2018;16(1):56

35. Sharma S, Palanchoke J, Abbott JH. Cross-cultural adaptation and validation of the Nepali translation of the patient-specific functional scale. J Orthop Sports Phys Ther. 2018;48(8):659-64

36. Prakash V, Shah S, Hariohm K: Cross-cultural adaptation of patient-reported outcome measures: a solution or a problem? Ann Phys Rehabil Med. 2019; 62(3):174-77.

37. Conway K, Acquadro C, Patrick DL. Usefulness of translatability assessment: results from a retrospective study. Qual Life Res. 2014;23(4):1199-210.

38. Eremenco SL, Cella D, Arnold BJ. A comprehensive method for the translation and cross-cultural validation of health status questionnaires. Eval Health Prof. 2005;28(2):212-32.

39. Terwee CB, Prinsen C, Chiarotto A, de Vet H, Bouter LM, Alonso J, Westerman MJ, Patrick DL, Mokkink LB. COSMIN methodology for assessing the content validity of PROMs-user manual; 2018.

40. Terwee CB, Prinsen CAC, Chiarotto A, Westerman MJ, Patrick DL, Alonso J, Bouter LM, de Vet HCW, Mokkink LB. COSMIN methodology for evaluating the content validity of patient-reported outcome measures: a Delphi study. Qual Life Res. 2018:27(5):1159-70.

41. Mokkink LB, Terwee CB, Knol DL, Stratford PW, Alonso J, Patrick DL, Bouter $L M$, de Vet HC. The COSMIN checklist for evaluating the methodological quality of studies on measurement properties: a clarification of its content. BMC Med Res Methodol. 2010;10:22

42. Central Bureau of Statistics. National population and housing census 2011. In: National Planning Commission Secretariat, Central Bureau of Statistics; 2012

43. Sharma S, Palanchoke J, Reed D, Haxby Abbott J. Translation, cross-cultural adaptation and psychometric properties of the Nepali versions of numerical pain rating scale and global rating of change. Health Qual Life Outcomes. 2017;15(1):236.

44. Sharma S, Pathak A, Jensen MP. Words that describe chronic musculoskeletal pain: implications for assessing pain quality across cultures. J Pain Res. 2016:9:1057-66.

45. Dworkin RH, Turk DC, Revicki DA, Harding G, Coyne KS, Peirce-Sandner S, Bhagwat D, Everton D, Burke LB, Cowan P, et al. Development and initial validation of an expanded and revised version of the short-form McGill pain questionnaire (SF-MPQ-2). Pain. 2009;144(1-2):35-42

46. Neblett R, Hartzell MM, Mayer TG, Cohen H, Gatchel RJ. Establishing clinically relevant severity levels for the central sensitization inventory. Pain Pract. 2017;17(2):166-75.

47. Cicchetti DV. Guidelines, criteria, and rules of thumb for evaluating normed and standardized assessment instruments in psychology. Psychol Assess. 1994;6(4):284-90.

48. de Vet HCW, Terwee CB, Mokkink LB, Knol DL. Measurement in medicine. Cambridge: Cambridge University Press; 2011.

49. Bland JM, Altman DG. Statistical methods for assessing agreement between two methods of clinical measurement. Lancet. 1986;1(8476):307-10.

50. Mokkink LB, Terwee CB, Patrick DL, Alonso J, Stratford PW, Knol DL, Bouter LM, de Vet HC. The COSMIN study reached international consensus on taxonomy, terminology, and definitions of measurement properties for health-related patient-reported outcomes. J Clin Epidemiol. 2010;63(7):737-45.

51. Cohen J. A power primer. Psychol Bull. 1992;112(1):155-9.

52. Sharma S, Ferreira-Valente A, Williams A, Abbott JH, Pais-Ribeiro J, Jensen MP. Group differences between-countries and between-languages in painrelated beliefs, coping, and catastrophizing in chronic pain: a systematic review. Pain Med. 2020;0(0):1-16.

53. Pitance L, Piraux E, Lannoy B, Meeus M, Berquin A, Eeckhout C, Dethier V, Robertson J, Meeus M, Roussel N. Cross cultural adaptation, reliability and validity of the French version of the central sensitization inventory. Man Ther. 2016;25:e83-4.

54. Bilika P, Neblett R, Georgoudis G, Dimitriadis Z, Fandridis E, Strimpakos N, Kapreli E: Cross-cultural Adaptation and Psychometric Properties of the Greek Version of the Central Sensitization Inventory. Pain Pract. 2019;20(2):188-96.

55. Bid DD, Soni NC, Rathod PV. Content validity and test-retest reliability of the Gujarati version of the central sensitization inventory. Natl J Integrated Res Med 2016, 7(5):18-24.

56. Kc S, Sharma S, Ginn K, Almadi T, Subedi H, Reed D. Cross-cultural adaptation and measurement properties of the Nepali version of the DASH (disability of arm, shoulder and hand) in patients with shoulder pain. Health Qual Life Outcomes. 2019;17(1):51.

57. Mokkink LB, Terwee CB, Patrick DL, Alonso J, Stratford PW, Knol DL, Bouter LM, de Vet HC. The COSMIN checklist for assessing the methodological quality of studies on measurement properties of health status measurement instruments: an international Delphi study. Qual Life Res. 2010;19(4):539-49.

\section{Publisher's Note}

Springer Nature remains neutral with regard to jurisdictional claims in published maps and institutional affiliations.

Ready to submit your research? Choose BMC and benefit from:

- fast, convenient online submission

- thorough peer review by experienced researchers in your field

- rapid publication on acceptance

- support for research data, including large and complex data types

- gold Open Access which fosters wider collaboration and increased citations

- maximum visibility for your research: over $100 \mathrm{M}$ website views per year

At $\mathrm{BMC}$, research is always in progress.

Learn more biomedcentral.com/submissions 